\title{
Interplay between structural and electronic properties with the metal-insulator transition in $\mathrm{NdNiO}_{3}$ thin films
}

Y. Eren Suyolcu ${ }^{1}$, Katrin Fürsich ${ }^{2}$, Matthias Hepting ${ }^{2}$, Zhicheng Zhong ${ }^{2}$, Yi Lu ${ }^{2}$, Yi Wang ${ }^{3}$, Georg Christiani $^{2}$, Gennady Logvenov ${ }^{2}$, Philipp Hansmann ${ }^{2}$, Matteo Minola ${ }^{2}$, Bernhard Keimer ${ }^{4}$, Peter A. van Aken $^{3}$ and Eva Benckiser ${ }^{3}$

${ }^{1}$ Department of Materials Science and Engineering, Cornell University, Ithaca, NY, USA, New York, United States, ${ }^{2}$ Max Planck Institute for Solid State Research, Stuttgart, Germany, United States, ${ }^{3}$ Max Planck Institute for Solid State Research, Stuttgart, Germany, Germany, ${ }^{4}$ Max Planck Institute for Solid State Research, Stuttgart, Germany, Baden-Wurttemberg, Germany

Epitaxial growth of transition-metal oxides allows for the design of unique heterostructures and controlling electronic correlations and novel physical properties [1]. The magnetic and electronic correlations in rare-earth nickelates are sensitive to structural modifications induced by the substrate [2]. While bulk $\mathrm{NdNiO}_{3}$ (NNO) exhibits a metal-insulator transition (MIT) at approximately $200 \mathrm{~K}, T_{\text {MIT }}$ can be enhanced $>100 \mathrm{~K}$, when NNO films are grown on [101]-oriented $\mathrm{NdGaO}_{3}$ (NGO) substrates, due to the specific three-fold interconnectivity between the film and substrate octahedral network [3]. Aberration-corrected scanning transmission electron microscopy (STEM) enables not only revealing structural and chemical information about the interfaces, but also provides imaging the oxygen atomic columns, which is crucial for understanding the underlying physics of oxide heterostructures and interfaces.

In this work, we combine comprehensive high-resolution STEM investigations with electrical transport measurements and density functional theory (DFT) calculations to investigate the evolution of the MIT in NNO films as a function of film thickness and NGO substrate crystallographic orientation. STEM techniques including high-angle annular dark-field (HAADF) and annular bright-field (ABF) imaging, electron energy-loss spectroscopy (EELS), and energy-dispersive X-ray spectroscopy (EDXS) are employed using a JEOL JEM-ARM200F STEM equipped with a cold field-emission electron source, a probe $\mathrm{C}_{\mathrm{s}}$-corrector (DCOR, CEOS GmbH), a Gatan GIF Quantum ERS spectrometer and a large solidangle JEOL Centurio SDD-type EDXS detector. STEM and EELS spectrum imaging were performed at probe semi-convergence angles of $20 \mathrm{mrad}$ and $28 \mathrm{mrad}$, respectively. The collection angles for HAADF and ABF imaging were 75-310 mrad and 11-23 mrad, respectively. The O-O picker tool [4] is used for STEM image analyses.

The overall structural quality (Fig. 1a) of the defect-free film with coherent interfaces (Fig. 1b, Fig.2a) is confirmed via STEM-HAADF imaging, and atomic-resolution STEM-EELS mapping of Ni and Ga (Fig. $2 \mathrm{~b}, \mathrm{c})$ suggests that elemental intermixing at the interfaces is limited to one monolayer. Moreover, our investigations on samples with identical NNO thickness grown on different NGO substrate cuts demonstrate that orthorhombic (101) and (011) substrate facets play a critical role in tuning the transition temperature $T_{\text {MIT. }}$ The growth on [101]-oriented substrates generally results in a higher $T_{\mathrm{MIT}}$, and [011]oriented films tend to form structural defects and non-equilibrium phases. Detailed information about the local octahedral distortions (Fig. 2d) at NGO-NNO interfaces has been obtained from high-resolution 
STEM-ABF imaging [5]. The bond angle measurements across the interfaces demonstrate that the $B-\mathrm{O}-B$ bond angle remains constant within the experimental error and will be comprehensively discussed [6].

a

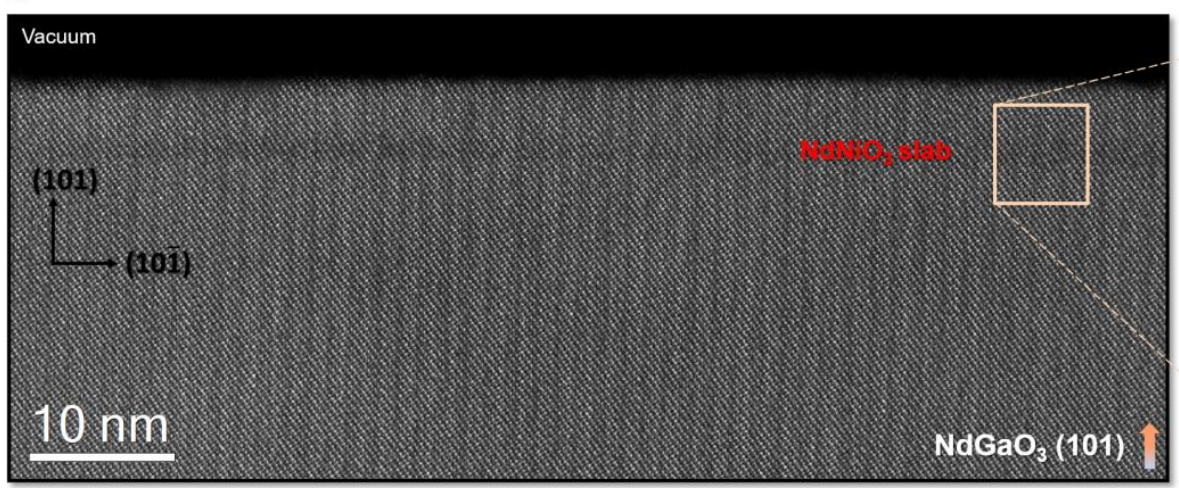

b

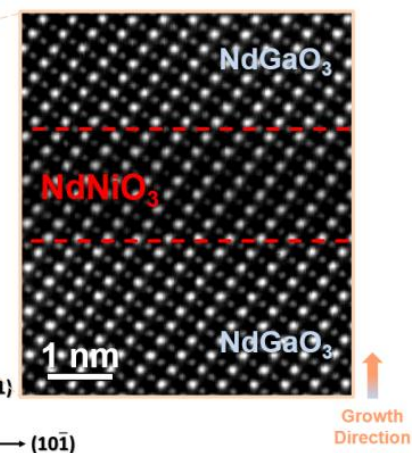

Figure 1. (a) Low-magnification STEM-HAADF image of a NNO film showing a high epitaxial quality. (b) Highmagnification STEM-HAADF image showing coherent interfaces and the NNO slab (darker contrast) sandwiched between the NGO substrate and NGO capping layer.
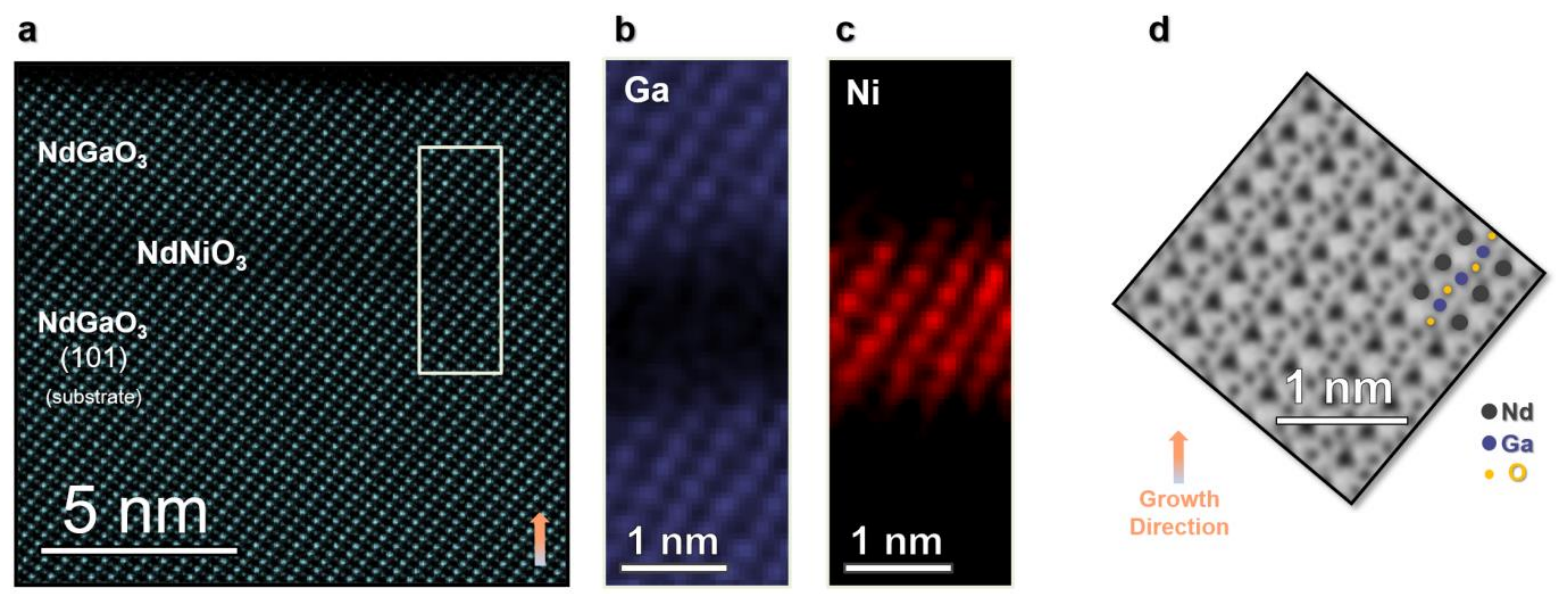

Figure 2. (a) STEM-HAADF image of the NNO slab (darker contrast) sandwiched between the NGO substrate and NGO capping layer. (b) Ga (purple) and (c) Ni (red) elemental maps obtained from STEMEELS spectrum imaging indicate the limited elemental intermixing at the interfaces. (d) STEM-ABF image taken from the NGO capping layer clearly shows the oxygen-column positions.

\section{References}

[1] Suyolcu, Y. E. et al., J. Supercond. Nov. Magn. 33 (2020), p. 107.

[2] Middey, S., et al., Annu. Rev. Mater. Res. 46 (2016), p. 305.

[3] Catalano, S., et al. APL Mater. 3 (2015), p. 062506.

[4] Wang, Y., et al., Ultramicroscopy 168 (2016), p. 46.

[5] Hepting, M., et al., Nat. Phys. 14 (2018), p 1097.

[6] Suyolcu, Y. E. et al., arXiv:2102.05415 (2021). 